\title{
Oncogenic RAS Generates Cancer Stem Cells in p53-Deficient Fibroblasts Through SOX2 Induced By CDK1-Mediated Protein 0-GlcNAcylation
}

Masahiro Shimizu

Nippon Medical School

Hiroshi Shibuya

Tokyo Medical and Dental University (TMDU)

Nobuyuki Tanaka ( $\square$ nobuta@nms.ac.jp )

Nippon Medical School

\section{Research Article}

Keywords: RAS, cancer stem cells, fibroblasts, SOX2, 0-GlcNAcylation

Posted Date: November 3rd, 2021

DOI: https://doi.org/10.21203/rs.3.rs-981157/v1

License: (c) (i) This work is licensed under a Creative Commons Attribution 4.0 International License.

Read Full License 


\section{Abstract}

Cancer stem cells (CSCs) have tumour initiation, self-renewal, and long-term tumour repopulation properties, and it is postulated that differentiated somatic cells can be reprogrammed to CSCs by oncogenic signals. We previously showed that oncogenic $H R A S^{V 12}$ conferred tumour initiation capacity in tumour suppressor p53-deficient $\left(p 53^{-/-}\right.$) primary mouse embryonic fibroblasts (MEFs) through transcription factor NF-kB-mediated enhancement of glucose uptake; however, the underlying mechanisms of RAS oncogene-induced CSC reprogramming have not been elucidated. Here, we found that the expression of the reprogramming factor SOX2 was induced by $H R A S^{V 12}$ in $p 53^{-/-}$MEFs. Moreover, gene knockout studies revealed that SOX2 is an essential factor for the generation of CSCs by $H R A S^{V 12}$ in mouse and human fibroblasts. We demonstrated that HRAS ${ }^{V 12}$-induced cyclin-dependent kinase 1 (CDK1) activity and subsequent enhancement of protein $O$-GlcNAcylation were required for SOX2 induction and CSC generation in these fibroblasts and cancer cell lines containing RAS mutations. Moreover, the CDK inhibitor dinaciclib and O-GIcNAcylation inhibitor OSMI1 reduced the number of CSCs derived from these cells. Taken together, our results reveal a signalling pathway and mechanism for CSC generation by oncogenic RAS and suggest the possibility that this signalling pathway is a therapeutic target for CSCs.

\section{Introduction}

Cancers are derived from cancer stem cells (CSCs), also known as cancer-initiating cells, that form tumours in vivo ${ }^{1}$. CSCs comprise a subset of tumour cells with tumour formation capacity, self-renewal properties, and long-term tumour repopulation activity ${ }^{2,3}$. The molecular mechanisms that support the reprogramming events leading to the generation of CSCs are considered to be similar to those of induced pluripotent stem cells (iPSCs), including the same regulatory factors and control mechanisms ${ }^{4}$. Indeed, reprogramming factors, including SRY-box 2 (SOX2), octamer-binding transcription factor 4 (OCT4, also known as POU5F1), Krüppel-like factor 4 (KLF4), and MYC (collectively known as OKSM transcription factors), and chromatin modifiers are required for establishment of iPSCs are associated with cancer generation ${ }^{4,5}$. It has been shown that transient expression of OKSM factors induced cancer development in various tissues in vivo and was accompanied by global changes in DNA methylation status ${ }^{6}$. Alterations of genes involved in the regulation of cell proliferation, such as oncogenes and tumour suppressor genes, may lead to cancer development ${ }^{7,8}$. Therefore, it is possible that analyses of reprogramming factors and cell growth regulators will lead to the elucidation of the mechanism of CSC generation. However, cancer develops because of the acquisition of successive hallmark cancer capabilities in a multistep pathogenic process ${ }^{7,8}$, and it is difficult to analyse the role of reprogramming factors in CSC generation using established cancer cell lines.

$P 53$ is the most frequently mutated gene in human cancer cells, and its gene product functions as a transcriptional activator of various target genes ${ }^{9}$. Accumulating evidence has shown that metabolic regulation by $\mathrm{p} 53$ is important for tumour suppression ${ }^{10,11}$. For example, p53 inhibits glycolysis, and the 
loss of p53 function changes the energy source from cellular respiration to glycolysis. This metabolic shift, known as the Warburg effect, favours tumour growth and is observed in many cancer cells ${ }^{12}$. In p53-deficient ( $p 53^{-/-}$) mouse embryonic fibroblasts (MEFs), we previously found that oncogenic cell transformation and enhanced aerobic glycolysis were induced by an activating mutant of HRAS $\left(\mathrm{HRAS}^{\mathrm{V} 12}\right.$ ) and were completely dependent on the transcription factor NF-KB ${ }^{13}$. Moreover, enhanced glycolysis-induced $O$-GlcNAcylation in $H R A S^{V 12}$-expressing $p 53^{-/-}$MEFs further activated NF-KB ${ }^{14}$, suggesting that the NF-KB/glycolysis activation loop in $p 53^{-/-}$cells is involved in enhanced energy production and CSC generation. The loss of p53 markedly increases the efficiency of somatic cell reprogramming to iPSCs, suggesting the existence of a p53-mediated barrier system that inhibits this reprogramming ${ }^{15-18}$. In various stem cell populations, enhanced glucose uptake is commonly observed and is critical for the acquisition and maintenance of stemness ${ }^{19}$. Moreover, direct 0 -GlcNAcylation of OCT4 and SOX2 has been shown to regulate pluripotency and reprogramming of embryonic stem cells ${ }^{20}$. In relation to these results, we found that IL-8 overexpression in colon and lung cancer cells enhanced $O$ GlcNAcylation, which was required for the production and maintenance of $\mathrm{CSCs}^{21}$. These results suggest that enhanced glycolysis and $O-$ GICNAcylation are involved in CSC generation and maintenance in p53deregulated cells.

Gain of function mutations in RAS genes are frequently found in human cancers and represent markers of poor prognosis in patients with certain cancers ${ }^{22,23}$. The $R A S$ family in humans comprises three genes, which are HRAS, NRAS, and KRAS. The proteins function as small GTPases. The activation of RAS is tightly regulated by guanine nucleotide exchange factors, which promote GTP-bound active states, and GTP-bound RAS interacts with and activates effector enzymes, such as RAF kinases. In the RAS-RAF cascade, activated RAF kinase phosphorylates and activates mitogen-activated protein kinase kinases 1 and 2 (MEK1 and MEK2), which leads to phosphorylation and activation of extracellular signal-regulated kinase (ERK) 1 and 2, also known as mitogen-activated protein kinase (MAPK) 3 and 1, respectively. The RAS-RAF-MAPK pathway mediates various biological functions, such as cell proliferation, survival, and differentiation, through a large number of ERK $1 / 2$ substrates that include transcription factors and cell cycle regulators ${ }^{24}$. Oncogenic mutations in the RAS-RAF-MAPK pathway contribute to various malignant phenotypes that include invasion, metastasis, relapse, and angiogenesis ${ }^{5}$.

Studies have shown that RAS-MAPK signalling promotes stemness and maintenance of $\mathrm{CSCs}^{25}$, but the underlying mechanisms have not been completely elucidated. Based on these studies, we analysed the expression of reprogramming factors in oncogenic HRAS-expressing $p 53^{-/-}$MEFs as a simple model system to understand the molecular mechanism of RASinduced CSC generation. As a result, we found that SOX2 induction by CDK1-mediated protein $O$-GIcNAcylation was essential for generation and maintenance of CSCs in p53-deficient cells.

\section{Results}


Oncogenic RAS induces SOX2-initiated CSC generation. Previously, we reported that oncogenic HRAS (HRASG12V mutant: HRAS ${ }^{V 12}$ ) induced tumorigenic properties in $p 53^{-/-} \mathrm{MEFs}^{26}$, suggesting that $H R A S^{V 12}$ promoted the generation of CSCs. To confirm this hypothesis, we generated $H R A S^{V 12}$-expressing $p 53^{-/-}$MEFs (Fig. 1A) and demonstrated that these cells formed tumours in nude mice (Fig. 1B). Previous studies have shown that CSCs display tumorigenic activity, and cancer cells with CSC-like properties will form spheres in low attachment culture conditions in medium containing growth factors ${ }^{27,28}$. As shown in Fig. $1 \mathrm{C}$, approximately $0.4 \%$ of $H R A S^{V 12}$-expressing $p 53^{-/-} \mathrm{MEFs}$ developed spheres in low attachment plates. It has been reported that the reprogramming factors OCT4, KLF4, and SOX2 are important for generation and maintenance of CSCs and are used as CSC markers in various cancers ${ }^{29-31}$. Therefore, we analysed the mRNA expression levels of these factors and found that the sphere-forming cells exhibited enhanced expression of OCT4, KLF4, and SOX2 compared with that of parental adherent cells (Fig. 1D). These results strongly suggested that sphere-forming cells from $H R A S^{V 12}$-expressing $p 53^{-/}$ -MEFs were CSCs. We also compared expression levels of these genes between adherent HRAS V12_ expressing $p 53^{-/-}$MEFs cells and $p 53^{-/-}$MEFs control cells. Interestingly, although OCT 4 expression was unchanged, the expression of SOX2 was markedly enhanced in HRAS ${ }^{V 12}$-expressing $p 53^{-/-}$MEFs cells (Fig. 1E and F). These results suggest that the expression of SOX2 as well as KLF4 was induced by $H R A S^{V 12}$ expression in $p 53^{-/-} \mathrm{MEFs}$, and OCT4 was induced in the process of CSC reprogramming.

It has been shown that tumorigenic properties are efficiently induced by co-expression of oncogenes in primary rodent cells but not in human cells, suggesting a mechanistic difference between human and rodent oncogenesis ${ }^{32}$. As previously shown, $H R A S^{V 12}$ and simian virus 40 large $T$ antigen (SV4O LT), which functionally inactivates p53 and the retinoblastoma tumour suppressor protein, were unable to induce tumorigenic properties in human cells ${ }^{32}$; SOX2 expression and sphere-forming activity were not induced by $p 53$ knockout $(\mathrm{KO})$ or $H R A S^{V 12}$ expression in the human lung fibroblast cell line TIG-3 (Supplementary Fig. S1 online). However, TIG-3 cells were transformed by the combination of SV40 T-ag, C-MYC, and HRAS ${ }^{V 12}$ (TIG-3-SMR cells) ${ }^{33,34}$, and sphere-forming cells were present in the TIG-3-SMR cell population (Supplementary Fig. S1C online). Notably, SOX2 expression was also elevated in TIG-3-SMR cells when compared with control TIG-3 cells (Supplementary Fig. S1D online). These results suggest that the induction of SOX2 by oncogenic signalling is regulated differently between mice and humans. However, the difference in tissue origin cannot be ruled out.

SOX2 expression is essential for HRAS ${ }^{\mathrm{V} 12}$-induced development of CSC properties. Studies have shown that SOX2 functions as an oncogene, and expression is closely related to metastasis and relapse of many cancers $^{35,36}$. Therefore, from these studies and the above results, it was possible that SOX2 functioned as an initiation factor for CSC reprogramming. To test this hypothesis, we deleted the SOX2 gene in $H R A S^{V 12}$-expressing $p 53^{-/-}$MEFs with three independent gRNAs using the CRISPR-Cas9 gene knockout system. Single cell-derived SOX2 KO clones from each gRNA were isolated, and immunoblotting and quantitative PCR (qPCR) showed a lack of SOX2 expression in each clone (Supplementary Fig. S2A, B online). Inhibition of SOX2 expression was reported to attenuate cancer cell proliferation ${ }^{37}$. SOX2 KO cells 
showed suppressed cell growth properties; however, proliferation was not completely inhibited (Fig. 2A). Next, we analysed the effect of SOX2 KO on the CSC properties found in $H R A S^{V 12}$-expressing $p 53^{-1}$ -MEFs. The appearance of sphere-forming cells and an increase in anchorage-independent colonyforming cells correlate with tumourigenicity ${ }^{38}$; however, these properties were not observed after SOX $2 \mathrm{KO}$ (Fig. 2B and Supplementary Fig. S2C online). Furthermore, we analysed the effect of SOX2 KO on tumourinitiating activity in vivo. HRAS V12-expressing $p 53^{-/-} \mathrm{MEF}$ s with or without SOX2 KO were subcutaneously injected into nude mice and tumour growth was monitored. Consistent with data presented in Fig. 1B, we confirmed tumour progression in mice injected with the $H R A S^{V 12}$-expressing $p 53^{-/-}$MEFs (Fig. 2C). However, SOX2 KO in these cells completely inhibited tumour development (Fig. 2C, D). Because the cell growth rate was suppressed in vitro by SOX2 KO (Fig. 2A), we measured tumorigenic activity for an extended period; however, we did not detect tumour formation up to 13 weeks after injection (Fig. 2C). Moreover, forced expression of SOX2 in $p 53^{-/-} \mathrm{MEFs}$ and $p 53^{-/-}$TIG-3 cells promoted sphere formation and tumour development (Supplementary Fig. S2D-H online). These results suggest that SOX2 expression is required for CSC generation in p53-deficient cells.

\section{SOX2 expression is induced by the RAF-MAPK pathway in HRAS ${ }^{\mathrm{V} 12}$-expressing $\mathrm{p} 53^{-/-}$MEFs. RAS} proteins directly activate the downstream effectors RAF and PI3K followed by the downstream activation of MAPK and AKT pathways, respectively ${ }^{24,39}$. Therefore, we next examined whether these effectors were involved in promoting SOX2 expression. Constitutively active forms of RAF (BRAF ${ }^{\mathrm{V} 600 \mathrm{E}}$ ) and PI3K (PI3K ${ }^{\text {CAAX }}$ ) were stably expressed in $p 53^{-/-}$MEFs. Although the increase in SOX2 mRNA expression was relatively weakly in PI3KCAAX -expressing cells, the levels of SOX2 and KLF4 mRNA in BRAF ${ }^{V 600 E_{-}}$ expressing cells were similar to that in HRAS ${ }^{V 12}$-expressing cells (Fig. $3 \mathrm{~A}$ ). These results suggest that $H R A S^{V 12}$ induces the expression of SOX2 mRNA through RAF and its downstream effectors MEK/ERK. Furthermore, the expression of HRAS ${ }^{\mathrm{V} 12}$ enhanced the activating phosphorylation of ERK and both the mRNA and protein expression of SOX2. These enhancements were significantly reduced by the MEK inhibitor U0126 but not by the PI3K inhibitor LY294002 (Fig. 3B-D). Similar results were obtained after treatment with the MEK inhibitor PD184352 (Fig. 3E). Immunofluorescent staining confirmed that the number of SOX2-positive cells was reduced by the MEK inhibitor but not by the PI3K inhibitor (Fig. 3F). Additionally, the number of sphere-forming cells was reduced after treatment of $H R A S^{V 12}$-expressing $p 53^{-/-}$MEFs with the MEK inhibitors (Fig. 3G). These results suggest that the RAF/MEK/ERK pathway is required for SOX2 induction and reprogramming of normal fibroblasts to CSCs.

CDK1 activity is required for SOX2 induction and generation of CSCs in RAS-activated cells. Activation of ERK enhances expression of cyclin D1, which binds to and activates cyclin-dependent kinase 4 and 6 (CDK4/6) during the $\mathrm{G} 1$ phase of the cell cycle ${ }^{40}$. Aberrant regulation of the CDK4/6-cyclin D1 axis is associated with the development of metastatic melanoma and breast cancer ${ }^{41,42}$. From these findings, it is possible that HRAS 12 induced SOX2 expression through ERK-mediated CDK4/6 activation. To test this possibility, we analysed the effect of the CDK4/6 inhibitor palbociclib ${ }^{43}$ on SOX2 expression and confirmed that the expression of SOX2 mRNA and protein levels were slightly reduced in HRAS ${ }^{V 12}$ 
expressing $p 53^{-/-}$MEFs after treatment with a high-dose of palbociclib (Supplementary Fig. S3A, B online). Similar results were obtained with TIG-3-SMR cells (Supplementary Fig. S3C online). These results suggest that CDK4/ 6 activity alone is insufficient for SOX2 induction. In addition to CDK4/6, the BRAF/MEK/MAPK pathway is also essential for some functions of CDK1 and CDK2 ${ }^{44-46}$. Recent studies have reported a role for CDK1 and CDK2 in the maintenance of stem cell pluripotency and CSC-like properties in breast cancer ${ }^{47-49}$. These reports suggested that CDK1 and/or CDK2 are required for SOX2 induction. Therefore, we examined the effects of the CDK1/2 inhibitors dinaciclib ${ }^{50}$ and roscovitine $e^{51}$ on SOX2 expression. We found that SOX2 mRNA and protein expression was strongly suppressed by dinaciclib (Fig. 4A, B) and roscovitine (Supplementary Fig. S3D, E online) in HRAS V12-expressing $p 53^{-/-}$ MEFs. Similar results for dinaciclib were observed in TIG-3-SMR cells (Fig. 4C). Moreover, the number of sphere-forming cells in $H R A S^{V 12}$-expressing $p 53^{-/-}$MEFs was largely suppressed by dinaciclib but only partially suppressed by palbociclib (Fig. 4D), which correlated with suppression of SOX2 expression levels (Fig. 4A and Supplementary Fig. S3A online). To further analyse whether CDKs were essential for SOX2 expression, we analysed SOX2 levels in HRAS ${ }^{V 12}$-expressing $p 53^{-/-}$MEFs after knockdown of $C D K 1$ or $C D K 2$ using siRNA. Immunoblot analysis showed that $C D K 1$, but not $C D K 2$, knockdown reduced the expression level of SOX2 protein (Fig. 4E). Treatment with dinaciclib, U0126, and PD184352, which suppressed the expression of SOX2 in this study, reduced the phosphorylation of $\mathrm{CDK} 11^{\mathrm{T} 161}$, an indicator of CDK1 activation ${ }^{52}$ (Supplementary Fig. S4A-C online). These results indicate that CDK1 activity is required for SOX2 expression in $H R A S^{V 12}$-expressing $p 53^{-/-}$MEFs. Additionally, we analysed the effect of dinaciclib on human cancer cell lines, including KRAS-mutated colon cancer (HCT116, SW480, and DLD1) and lung cancer (H460 and A549) cells. Dinaciclib suppressed SOX2 mRNA expression in these cancer cells (Fig. 4F) and the number of sphere-forming cells from the HCT116, SW480, and H460 lines (Fig. 4G). These results suggest that CDK1-mediated SOX2-induction promotes the generation of CSCs in human cancer cells with RAS mutations.

\section{Enhanced 0-GIcNAc modification induced by the RAS/MAPK/CDK1 pathway is required for SOX2 protein expression and generation of CSCs. O-GICNAcylation is the post-translational addition of $\mathrm{N}$ -} acetylglucosamine (also known as $\mathrm{O}$-GIcNAc) to serine or threonine residues of proteins that contributes to stability and activity of the modified protein. $O$-GIcNAcylation is a reversible modification mediated by $O$-GIcNAc transferase (OGT) and O-GIcNAcase (OGA); OGT catalyses the addition of $O$-GIcNAc to the target protein, whereas OGA removes $O$-GIcNAc from proteins by hydrolysis ${ }^{53}$. Previously, we reported that enhanced $O$-GICNAcylation is important for SOX2 expression and maintenance of CSC properties, including sphere- and tumour-forming activities, in colon and lung cancer cells ${ }^{21}$. These findings suggested the possibility that $O$-GICNAC modifications are involved in acquisition of CSC properties. Therefore, we determined the $O$-GIcNAc levels in HRAS ${ }^{V 12}$-expressing $p 53^{-/-}$MEFs and TIG-3-SMR cells and found elevated levels of protein $O$-GIcNAcylation compared with those in the respective control cells (Fig. 5A, B). Next, we analysed the cells after treatment with OSMI1, a cell-permeable, small molecule OGT inhibitor ${ }^{37}$. OSMI1 treatment suppressed total O-GIcNAcylation levels of proteins and SOX2 expression in these cells (Fig. 5A, B). In contrast, treatment of HRAS ${ }^{V 12}$-expressing $p 53^{-/-} \mathrm{MEFs}$ with thiamet $\mathrm{G}$, a 
specific OGA inhibitor that increases $O$-GIcNAcylation ${ }^{38}$, enhanced total $O$-GIcNAcylation levels and SOX2 expression (Fig. 5C). Interestingly, the OSMI1-mediated reduction in SOX2 levels was attenuated by treatment with the proteasome inhibitor MG-132 (Fig. 5A, B). The mRNA levels of SOX2 in HRAS ${ }^{V 12}$ expressing $p 53^{-/-}$MEFs were not significantly changed by OSMI1 and thiamet G (Fig. 5D), indicating that O-GlcNAcylation regulated SOX2 expression at the post-transcriptional level. Consistent with these results, the numbers of sphere-forming cells decreased and increased after treatment with OSMI1 and thiamet G, respectively (Fig. 5E). These results suggest that increased $O$-GlcNAcylation is required for SOX2 protein expression and sphere-forming activity in these cells.

Finally, we analysed the role of RAS/MAPK-activated CDK1 in the induction of 0 -GIcNAcylation. The elevated levels of $O$-GIcNAcylation in HRAS $S^{V 12}$-expressing $p 53^{-/-}$MEFs and TIG-3-SMR cells were suppressed by dinaciclib in a dose dependent manner (Supplementary Fig. S4A, B online). The MEK inhibitors U0126 and PD184352 reduced the phosphorylation of $\mathrm{CDK} 1^{\mathrm{T} 161}$ and $\mathrm{O}$-GIcNAcylation levels in $H R A S^{V 12}$-expressing $p 53^{-/-}$MEFs (Supplementary Fig. S4C online). Furthermore, knockdown of CDK1, but not CDK2, with siRNA inhibited O-GIcNAcylation levels in these cells (Supplementary Fig. S4D online). In contrast, treatment with palbociclib had no effect on O-GlcNAcylation levels (Supplementary Fig. S3B, C online). In addition, SOX2 expression (as shown in Fig. 4F, G) and O-GIcNAcylation levels in KRASactivated cancer cells were suppressed by dinaciclib (Fig. 5F). These results suggest that RAS/RAF/MAPK pathway-induced CDK1 activation is important for induction of $O$-GlcNAcylation, and this activation pathway is required for SOX2 expression and subsequent CSC generation.

\section{Discussion}

Accumulating evidence has revealed that serial oncogenic mutations in stem cells and even in differentiated somatic cells may induce the generation of $\mathrm{CSCs}^{54-58}$. The dedifferentiation of somatic cells into CSCs is thought to be induced by a reprogramming mechanism similar to that observed in the production of iPSCs ${ }^{59,60}$. Viewed through the "hallmarks of cancer" 7,8 , CSCs may be generated by iPSC reprogramming factors that are induced by gain-of-function oncogenic and loss-of-function tumour suppressor gene mutations. Therefore, the HRAS $S^{V 12}$-expressing $p 53^{-/-} \mathrm{MEFs}$ provided a simple and easyto-analyse model for evaluating the signalling system used by oncogenes to regulate reprogramming factors. In our previous studies, we found that the $H R A S^{V 12}$ mutation conferred tumour-initiating activity, a hallmark of CSCs, in $p 53^{-/-} \mathrm{MEFs}^{26}$, and this phenomenon was completely dependent on NF-KBinduced aerobic glycolysis ${ }^{13}$. In this study, we found that $H R A S^{V 12}$ induced MAPK-CDK1 signal-dependent induction of SOX2 mRNA transcription and $O$-GIcNAcylation-mediated SOX2 protein accumulation. Although the transcriptional induction mechanism of SOX2 by CDK1 was not elucidated in this experimental system, CDK1-mediated induction of SOX2 has been analysed in other studies. For example, it was reported that CDK1-induced phosphorylation directly activated transcription factor CP2-like protein

1 (TFCP2L1), which is an activator of pluripotency-associated genes, including SOX $2^{61}$. CDK1 
phosphorylated and inhibited the histone lysine demethylase KDM5B, which is a transcriptional suppressor of the pluripotency genes SOX2 and $N A N O G^{62}$.

It has been shown that the hexosamine biosynthetic pathway shunts glycolysis toward the production of a key substrate for 0 -GIcNAcylation and is activated by enhanced glycolysis ${ }^{63}$. The RAS/MAPK pathway induces metabolic reprogramming, including enhanced glycolysis ${ }^{64}$. Therefore, in addition to NF-KBinduced enhancement of glycolysis in $p 53^{-/-} \mathrm{MEFs}^{13}$, our present results suggest that the RAS/MAPK/CDK1 pathway further promoted glycolysis and resulted in enhanced protein $O$ GIcNAcylation. Moreover, we also found that $O$-GIcNAcylation inhibited proteasomal degradation of SOX2, suggesting that direct $O$-GIcNAcylation of SOX2 or other factor(s) is involved in the ubiquitinmediated degradation system. However, in our previous report and in the present study, we were unable to detect direct $O$-GIcNAcylation of SOX2 as demonstrated in pluripotent cells ${ }^{65}$. Although SOX2 expression was induced by mRNA levels in transformed cells (Fig. 1E), we found that $O$-GlcNAc modification regulated SOX2 at post-transcriptional levels (Fig. 5A-D). For this issue, we indicated that deletion of SOX2 gene in $H R A S^{V 12}$-expressing $p 53^{-/-}$MEFs reduced O-GIcNAc levels (Supplementary Fig. S4E online), and exogenous SOX2 expression induced protein 0 -GlcNAcylation in $p 53^{-/-}$MEFs (Supplementary Fig. S4F online). These data suggest that transcriptionally elevated SOX2 expression promotes $O$-GlcNAc levels, and enhanced $O$-GIcNAcylation could promote expression of SOX2 at posttranscriptional levels.

The tumour suppressor protein p53 is referred to as "the guardian of the genome" because it continuously surveys damaged genomic DNA and facilitates DNA repair ${ }^{66}$. Moreover, in response to oncogenic signalling, the p53 tumour surveillance system eliminates cells through p53-mediated apoptosis or induction of senescence ${ }^{67}$. In addition to these functions, accumulating evidence has indicated that regulation of cellular metabolism by $p 53$ is involved in tumour suppression ${ }^{68}$. It was demonstrated that mice expressing acetylation-defective p53 mutants lost the ability to induce apoptosis and senescence but retained tumour suppressive function and the ability to modulate the expression of metabolic genes $^{69}$. In $p 53^{-/-}$MEFs, we previously found that oncogenic transformation by $H R A S^{V 12}$ was dependent on enhanced aerobic glycolysis through NF-KB-mediated induction of the glucose transporter GLUT3 ${ }^{13}$. In this previous study, we found that GLUT3 (also known as SLC2A3) functioned as an oncogene because $p 53^{-/-}$MEFs underwent oncogenic transformation after overexpression of GLUT3 without HRAS ${ }^{V 12}$. These results suggested that enforced glucose flux induced CSC generation in cells that lacked p53 function. Furthermore, we found that $O$-GlcNAcylation increased with accelerated glucose consumption in $p 53^{-/-}$MEFs and was further enhanced by $H R A S^{V 12}$ expression ${ }^{14}$. In the present study, CSC generation in $H R A S^{V 12}$-expressing $p 53^{-/-}$MEFs was completely dependent on the induction of SOX2, and enhancement of $O$-GICNAcylation by the HRAS ${ }^{12}$-MAPK-CDK1 pathway was important for SOX2 induction. Therefore, it is possible that glycolysis may be hampered by $\mathrm{p} 53$, which prevents excessive $O$ GIcNAcylation-mediated induction of SOX2. Indeed, it has been shown that oncogenes, such as HRAS ${ }^{V 12}$, activate p53 in untransformed cells ${ }^{67}$, and activation of p53 results in inhibition of glucose consumption 
and glycolysis ${ }^{13,70}$. Moreover, it has been reported that the OKSM reprogramming factors induce p53mediated senescence ${ }^{71}$, suggesting the possibility that these reprogramming factors limit their own function using p53-mediated negative feedback. Therefore, at least in MEFs, p53 may exert a tumour suppressor function through the regulation of $O$-GIcNAcylation by limiting glucose metabolism.

In MEFs, induction of SOX2 was essential for CSC reprogramming by HRAS ${ }^{V 12}$, but this pathway may not be the only signal for CSC generation. For example, in the context of inflammation-induced cancer, we found that the activated form of MYD88, a regulator of inflammatory signalling, promoted CSC generation in $p 53^{-/-}$MEFs through activation of the NF-KB-HIF1-OCT4, but not the SOX2, induction pathway $^{72}$. Furthermore, in the present study, expression of $H R A S^{V 12}$ alone was insufficient to induce SOX2 in human TIG-3 cells. Although it is possible that this difference was because of cell type specificity, differences between human and rodent cells cannot be ruled out. In contrast to human cells, primary rodent cells are efficiently converted to tumorigenic cells by the co-expression of oncogenes, suggesting a fundamental difference between humans and rodents ${ }^{32}$. Therefore, these results suggest the possibility that an unidentified tumour suppressive mechanism other than p53 exists in humans and prevents the induction of SOX2 by RAS. Further analyses are required to clarify this mechanism.

\section{Materials And Methods}

\section{Cell culture and reagents}

The $p 53^{-/-}$MEFs, HRAS ${ }^{V 12}$-expressing $p 53^{-/-}$MEFs, TIG-3, and TIG-3-SMR cells were prepared as previously described ${ }^{13,14}$. These cells were cultured in DMEM (Nissui, Japan) containing $10 \%$ foetal bovine serum (FBS). HCT116, SW480, and DLD1 colon cancer cell lines and HCC827 and H460 lung cancer cell lines were purchased from ATCC (Manassas, VA, USA). HCT116 cells were cultured in McCoy's 5A medium (Gibco, Waltham, MA, USA), SW480 and DLD1 cells were grown in DMEM, and HCC827 and H460 cells were cultured in RPMI 1640 medium (Nissui, Japan), each containing 10\% FBS. All cells were tested for mycoplasma contamination using the MycoAlert ${ }^{\text {TM }}$ Mycoplasma Detection Kit (Lonza, Switzerland), which confirmed the absence of mycoplasma. U0126 (Promega, Madison, WI, USA), LY294002 (Fujifilm Wako, Japan), PD184352, dinaciclib, palbociclib (Selleck Biotech, Houston, TX, USA), thiamet G (Sigma-Aldrich, St. Louis, MO, USA), and OSMI1 (Cayman Chemical, Ann Arbor, MI, USA) were used in the inhibition experiments. MG-132, and anti-c-H-Ras (OP23) and anti-SV40 T antigen (DP01) antibodies were obtained from Calbiochem (Merck, Germany). Anti-OGT (H-300), anti-TERT (H-231), and anti-c-Myc (9E10) antibodies were obtained from Santa Cruz Biotechnology (Dallas, TX, USA). Anti-OGlcNAc antibody (CTD110.6) was purchased from Covance (Princeton, NJ, USA). Anti-SOX2 (D9B8N), anti-ERK1/2 (\#9102), and anti-pERK1/2 ${ }^{\mathrm{T} 202 / Y 204}$ (\#9101) antibodies were purchased from Cell Signaling Technology (Danvers, MA, USA). Anti-CDK1 (A2861), anti-pCDK1 ${ }^{\mathrm{T161}}$ (AP0324), and anti-CDK2 (A0294) antibodies were obtained from ABclonal (Cambridge, MA, USA). Anti- $\beta$-actin (AC-74) was obtained from Sigma-Aldrich. All antibodies were used at a 1:1000 dilution. 


\section{Expression vectors and virus infection}

A SOX2 sgRNA CRISPR/Cas9 All-in-One Lentivirus vector set (Applied Biological Materials, Canada) was used for knockout of the SOX2 gene. The sgRNA sequences were as follows: sgRNA\#1, CAACCAGAAGAACAGCC; sgRNA\#2, TCATCGACGAGGCCAAG; and sgRNA\#3, ATTATAAATACCGGCCG. For knockout of the $p 53$ gene in TIG-3 cells, the lentivirus vector pSpCas9(BB)-2A-Puro (PX459) V2.0 was obtained from the Addgene repository (plasmid \#62988, Cambridge, MA, USA), and the target sequence for $p 53$ (ACCAGCAGCTCCTACACCGG) was cloned into the PX459 vector following the repository's recommended Zhang lab protocol. Retroviral vectors pMXs-mSOX2-IP (plasmid \#15919), pBabe $\mathrm{BRAF}^{\mathrm{V} 600 \mathrm{E}}$ (plasmid \#17544), and pBabe PI3K p110 CAAX (plasmid \#13339) containing the puromycin resistance marker were purchased from Addgene. The pBabe SV40 T antigen, pBabe c-Myc, and pBabe HRAS $^{V 12}$ vectors were used with blasticidin, neomycin, and hygromycin selectable markers, respectively. Lentiviral and retroviral infections were performed as previously described ${ }^{23,64}$. The infected cells were selected with the appropriate antibiotics.

\section{siRNA and transfection}

The predesigned short interfering RNA (siRNA) for mouse Cdk1 and Cdk2 were obtained from SigmaAldrich: siCDK1\#1, SASI_Mm01_00179321; siCDK1\#2, SASI_Mm01_00179322; siCDK2\#1, SASI_Mm02_00323492; siCDK2\#2, SASI_Mm01_00151932. We performed reverse transfection of siRNA using Lipofectamine ${ }^{T M}$ RNAiMAX Transfection Reagent (Thermo Fisher Scientific, Waltham, MA, USA).

\section{Immunoblot analysis}

Cells were lysed with TNE buffer (10 mM Tris-HCl, pH 7.4; 1\% NP-40; 150 mM NaCl; 1 mM EDTA; 1 mM DTT, and protease inhibitor cocktail) (Nacalai Tesque, Japan). Protein concentrations were measured using the Bradford assay. Cellular proteins $(20 \mu \mathrm{g})$ were separated using sodium dodecyl sulfatepolyacrylamide gel electrophoresis and then transferred to polyvinylidene difluoride membranes (Merck). The membranes were probed with primary antibodies, followed by incubation with horseradish peroxidase-conjugated mouse or rabbit immunoglobulin G (GE Healthcare, England) and visualisation using Chemi-Lumi-One Super or Ultra assay kits (Nacalai Tesque). The protein bands were digitalised using the LAS-3000 mini image analyser (Fujifilm, Japan).

\section{Quantitative real-time PCR}

Total RNA was extracted using the NucleoSpin RNA kit (Macherey-Nagel, Germany) following the manufacturer's instructions. Double-stranded cDNA was prepared from total RNA using oligonucleotide (dT), random primers, and Superscript III reverse transcriptase (Invitrogen, Carlsbad, CA, USA). Quantitative real-time PCR (qPCR) analysis was performed as previously described ${ }^{23}$. The following probes were predesigned from Applied Biosystems ${ }^{\circledR}$ (Thermo Fisher Scientific): mouse $\beta$-actin, Mm00607939_s1; mouse Oct4, Mm03053917_g1; mouse KIf4, Mm00516104_m1; mouse Sox2, 
Mm03053810_s1; human $\beta$-actin, Hs00357333_g1; human OCT4, Hs03005111_m1; human KLF4, Hs00358836_m1; human SOX2, Hs01053049_s1; and human NANOG, Hs04260366_m1.

\section{Immunofluorescent analysis}

The $p 53^{-/-}$MEFs and HRAS $S^{V 12}$-expressing $p 53^{-/-}$MEFs $\left(4 \times 10^{4}\right)$ were cultured on glass coverslips in 6well plates and treated with U0126 $(10 \mu \mathrm{M})$ or LY294002 $(20 \mu \mathrm{M})$ for $24 \mathrm{~h}$. The cells were washed in phosphate-buffered saline (PBS), fixed with $4 \%$ paraformaldehyde in PBS for $15 \mathrm{~min}$, and permeabilised with $0.5 \%$ Triton X-100 in PBS for 10 min. Permeabilised cells were blocked with Blocking One Histo reagent (Nacalai Tesque) for $30 \mathrm{~min}$ and incubated with anti-SOX2 antibody for $1 \mathrm{~h}$ at room temperature. Alexa Flour 488-conjugated anti-rabbit antibody (Thermo Fisher Scientific) was used as the secondary antibody for $1 \mathrm{~h}$ at room temperature. VECTASHIELD Mounting Medium with DAPI (Vector Laboratories, Burlingame, CA, USA) was used to stain nuclei and for mounting the cells on slides. Images were acquired using a fluorescence microscope (BioZero BZ-8100; Keyence, Japan) and analysed with BZ Analyzer software (Keyence).

\section{Cell growth analysis}

HRAS $S^{V 12}$-expressing $p 53^{-/-}$MEFs $\left(1 \times 10^{5}\right)$ expressing each SOX2 sgRNA separately were seeded in 6well plates. Cell numbers were determined using a Vi-CELL cell analyser (Beckman, Brea, CA, USA) on the indicated days after plating, and cell growth curves were created.

\section{Sphere formation assay}

Cells $\left(5 \times 10^{3}\right.$ or $\left.1 \times 10^{4}\right)$ were plated in 6-well, ultra-low attachment plates and grown in serum-free DMEM/F12 medium containing epidermal growth factor $(20 \mathrm{ng} / \mathrm{mL})$ and basic fibroblast growth factor $(10 \mathrm{ng} / \mathrm{mL})$ for 7 days. The numbers of spheres were counted in each treatment group. Sphere images were captured using the BZ-8100 microscope.

\section{Colony formation assay}

A layer of $1.5 \%$ (weight/volume) agarose prepared in DMEM containing $10 \% \mathrm{FBS}$ was added to the wells of 6-well plates. Agarose (0.6\%) containing $3 \times 10^{4} \mathrm{HRAS}^{\mathrm{V} 12}$-expressing $p 53^{-/-} \mathrm{MEFs}$ containing each SOX2 sgRNA was added to the top of the first layer. After 30 days, each well was stained with $0.005 \%$ crystal violet (Sigma-Aldrich), and the colonies were counted.

\section{Animal experiments and cell line xenografts}

The animal experiment protocol was approved by the Ethics Committee on Animal Experiments of Nippon Medical School (ethics approval number 26-020, 27-188). Animal experiments were carried out in accordance with the guidelines for Animal Experiments of Nippon Medical School and the guidelines of The Law and Notification of the Government of Japan as well as the ARRIVE guidelines. Mice were maintained at $20-24{ }^{\circ} \mathrm{C}$ in a facility with a $12 \mathrm{~h} \mathrm{light/} \mathrm{dark} \mathrm{cycle} \mathrm{and} 40 \%-70 \%$ humidity. The mice were 
allowed free access to water and standard MF laboratory mouse chow (Oriental Yeast Co., Itd. Tokyo, Japan) and housed at a maximum number of five per cage. All mice were checked for stress each day. For the xenograft experiments, 5-week-old male BALB/cAJcl-nu/nu mice were purchased from CLEA Japan, Inc. (Tokyo, Japan) and assigned at random to the experiments. These mice were subcutaneously injected with $1 \times 10^{5}$ cells of $p 53^{-/-}$MEFs, HRAS ${ }^{V 12}$-expressing $p 53^{-/-}$MEFs or HRAS ${ }^{V 12}$-expressing $p 53^{-1}$ - MEFs containing SOX2 gRNA. The number of mice used are indicated for each experiment. Tumour growth was monitored every 3 days for 3 weeks and each week thereafter by caliper measurements, and tumour size was determined using the following formula: (Length $\times$ Width $\left.{ }^{2}\right) / 2$. At the end of the experiments ( 3 or 13 weeks post-injection), mice were euthanized by cervical dislocation, then each tumour were removed and weighed, and also collected for further experiments.

\section{Statistical analysis}

All experiments were repeated at least three times independently. Data are presented as means \pm standard deviation (SD). Statistical analysis was performed with the Student's $t$-test using Microsoft Excel (Microsoft, DC, USA). $P<0.05$ was considered statistically significant.

\section{Declarations}

\section{Acknowledgments}

We thank Y. Abe. Y. Nakajima, A. Tanimura, and I. Uehara for discussion, and M. Kajita, Y. Asano, M. Kawagoe, and T. Takatera for technical support. This work was supported by Grants-in-Aid for scientific research from the Ministry of Education, Science, Sports and Culture of Japan (MEXT/JSPS KAKEN Grant Number 17H04554 and 19K16782). We thank Susan Zunino, PhD, from Edanz (https://jp.edanz.com/ac) for editing a draft of this manuscript.

\section{Author contributions}

M.S., H.S., and N.T. designed the study, analysed the data, and wrote the manuscript. M.S. performed all of the experiments, and M.S. and N.T. analysed the data. M.S., H.S., and N.T. discussed the data.

\section{Data availability}

The data are available from the corresponding author upon request.

Competing interests: The authors declare that they have no competing interests.

\section{References}

1. Bjerkvig, R., Tysnes, B. B., Aboody, K. S., Najbauer, J. \& Terzis, A. J. Opinion: the origin of the cancer stem cell: current controversies and new insights. Nat Rev Cancer 5, 899-904, doi:10.1038/nrc1740 (2005). 
2. Batlle, E. \& Clevers, H. Cancer stem cells revisited. Nat Med 23, 1124-1134, doi:10.1038/nm.4409 (2017).

3. Lytle, N. K., Barber, A. G. \& Reya, T. Stem cell fate in cancer growth, progression and therapy resistance. Nat Rev Cancer 18, 669-680, doi:10.1038/s41568-018-0056-x (2018).

4. Goding, C. R., Pei, D. \& Lu, X. Cancer: pathological nuclear reprogramming? Nat Rev Cancer 14, 568573, doi:10.1038/nrc3781 (2014).

5. Flavahan, W. A., Gaskell, E. \& Bernstein, B. E. Epigenetic plasticity and the hallmarks of cancer. Science 357, doi:10.1126/science.aal2380 (2017).

6. Ohnishi, K. et al. Premature termination of reprogramming in vivo leads to cancer development through altered epigenetic regulation. Cel/ 156,663-677, doi:10.1016/j.cell.2014.01.005 (2014).

7. Hanahan, D. \& Weinberg, R. A. The hallmarks of cancer. Cel/ 100, 57-70, doi:10.1016/S00928674(00)81683-9 (2000).

8. Hanahan, D. \& Weinberg, R. A. Hallmarks of cancer: the next generation. Cel/ 144, 646-674, doi:10.1016/j.cell.2011.02.013 (2011).

9. Vogelstein, B., Lane, D. \& Levine, A. J. Surfing the p53 network. Nature 408, 307-310, doi:10.1038/35042675 (2000).

10. Labuschagne, C. F., Zani, F. \& Vousden, K. H. Control of metabolism by p53 - Cancer and beyond. Biochim Biophys Acta Rev Cancer 1870, 32-42, doi:10.1016/j.bbcan.2018.06.001 (2018).

11. Levine, A. J. p53: 800 million years of evolution and 40 years of discovery. Nat Rev Cancer 20, 471480, doi:10.1038/s41568-020-0262-1 (2020).

12. Koppenol, W. H., Bounds, P. L. \& Dang, C. V. Otto Warburg's contributions to current concepts of cancer metabolism. Nat Rev Cancer 11, 325-337, doi:10.1038/nrc3038 (2011).

13. Kawauchi, K., Araki, K., Tobiume, K. \& Tanaka, N. p53 regulates glucose metabolism through an IKKNF-kappaB pathway and inhibits cell transformation. Nat Cell Bio/ 10, 611-618, doi:10.1038/ncb1724 (2008).

14. Kawauchi, K., Araki, K., Tobiume, K. \& Tanaka, N. Loss of p53 enhances catalytic activity of IKKbeta through O-linked beta-N-acetyl glucosamine modification. Proc Natl Acad Sci U S A 106, 3431-3436, doi:10.1073/pnas.0813210106 (2009).

15. Hong, H. et al. Suppression of induced pluripotent stem cell generation by the p53-p21 pathway. Nature 460, 1132-1135, doi:10.1038/nature08235 (2009).

16. Li, H. et al. The Ink4/Arf locus is a barrier for iPS cell reprogramming. Nature 460, 1136-1139, doi:10.1038/nature08290 (2009).

17. Marion, R. M. et al. A p53-mediated DNA damage response limits reprogramming to ensure iPS cell genomic integrity. Nature 460, 1149-1153, doi:10.1038/nature08287 (2009).

18. Utikal, J. et al. Immortalization eliminates a roadblock during cellular reprogramming into iPS cells. Nature 460, 1145-1148, doi:10.1038/nature08285 (2009). 
19. Tsogtbaatar, E., Landin, C., Minter-Dykhouse, K. \& Folmes, C. D. L. Energy Metabolism Regulates Stem Cell Pluripotency. Front Cell Dev Bio/ 8, 87, doi:10.3389/fcell.2020.00087 (2020).

20. Jang, H. et al. O-GIcNAc regulates pluripotency and reprogramming by directly acting on core components of the pluripotency network. Cell Stem Cell 11, 62-74, doi:10.1016/j.stem.2012.03.001 (2012).

21. Shimizu, M. \& Tanaka, N. IL-8-induced O-GIcNAc modification via GLUT3 and GFAT regulates cancer

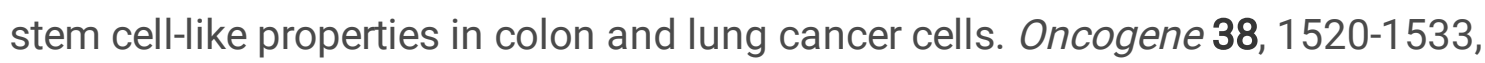
doi:10.1038/s41388-018-0533-4 (2019).

22. Prior, I. A., Lewis, P. D. \& Mattos, C. A comprehensive survey of Ras mutations in cancer. Cancer Res 72, 2457-2467, doi:10.1158/0008-5472.CAN-11-2612 (2012).

23. Pylayeva-Gupta, Y., Grabocka, E. \& Bar-Sagi, D. RAS oncogenes: weaving a tumorigenic web. Nat Rev Cancer 11, 761-774, doi:10.1038/nrc3106 (2011).

24. Simanshu, D. K., Nissley, D. V. \& McCormick, F. RAS Proteins and Their Regulators in Human Disease. Cell 170, 17-33, doi:10.1016/j.cell.2017.06.009 (2017).

25. Lavoie, H., Gagnon, J. \& Therrien, M. ERK signalling: a master regulator of cell behaviour, life and fate. Nat Rev Mol Cell Bio/ 21, 607-632, doi:10.1038/s41580-020-0255-7 (2020).

26. Tanaka, N. et al. Cellular commitment to oncogene-induced transformation or apoptosis is dependent on the transcription factor IRF-1. Cel/ 77, 829-839, doi:10.1016/0092-8674(94)90132-5 (1994).

27. Eramo, A. et al. Identification and expansion of the tumorigenic lung cancer stem cell population. Cell Death Differ 15, 504-514, doi:10.1038/sj.cdd.4402283 (2008).

28. Vermeulen, L. et al. Single-cell cloning of colon cancer stem cells reveals a multi-lineage differentiation capacity. Proc Natl Acad Sci U S A 105, 13427-13432, doi:10.1073/pnas.0805706105 (2008).

29. Bareiss, P. M. et al. SOX2 expression associates with stem cell state in human ovarian carcinoma. Cancer Res 73, 5544-5555, doi:10.1158/0008-5472.CAN-12-4177 (2013).

30. Qi, X. T. et al. KLF4 functions as an oncogene in promoting cancer stem cell-like characteristics in osteosarcoma cells. Acta Pharmacol Sin 40, 546-555, doi:10.1038/s41401-018-0050-6 (2019).

31. Wang, G., Zhou, H., Gu, Z., Gao, Q. \& Shen, G. Oct4 promotes cancer cell proliferation and migration and leads to poor prognosis associated with the survivin/STAT3 pathway in hepatocellular carcinoma. Oncol Rep 40, 979-987, doi:10.3892/or.2018.6491 (2018).

32. Hahn, W. C. et al. Creation of human tumour cells with defined genetic elements. Nature 400, 464468, doi:10.1038/22780 (1999).

33. Boehm, J. S., Hession, M. T., Bulmer, S. E. \& Hahn, W. C. Transformation of human and murine fibroblasts without viral oncoproteins. Mol Cell Bio/25, 6464-6474, doi:10.1128/MCB.25.15.64646474.2005 (2005). 
34. Hahn, W. C. et al. Enumeration of the simian virus 40 early region elements necessary for human cell transformation. Mol Cell Biol 22, 2111-2123, doi:10.1128/MCB.22.7.2111-2123.2002 (2002).

35. Xiang, R. et al. Downregulation of transcription factor SOX2 in cancer stem cells suppresses growth and metastasis of lung cancer. Br J Cancer 104, 1410-1417, doi:10.1038/bjc.2011.94 (2011).

36. Zheng, J. et al. Sox 2 modulates motility and enhances progression of colorectal cancer via the RhoROCK signaling pathway. Oncotarget 8, 98635-98645, doi:10.18632/oncotarget.21709 (2017).

37. Liu, P. et al. SOX2 Promotes Cell Proliferation and Metastasis in Triple Negative Breast Cancer. Front Pharmaco/ 9, 942, doi:10.3389/fphar.2018.00942 (2018).

38. Mori, S. et al. Anchorage-independent cell growth signature identifies tumors with metastatic potential. Oncogene 28, 2796-2805, doi:10.1038/onc.2009.139 (2009).

39. Downward, J. Targeting RAS and PI3K in lung cancer. Nat Med 14, 1315-1316, doi:10.1038/nm12081315 (2008).

40. Sherr, C. J., Beach, D. \& Shapiro, G. I. Targeting CDK4 and CDK6: From Discovery to Therapy. Cancer Discov 6, 353-367, doi:10.1158/2159-8290.CD-15-0894 (2016).

41. Bustos, M. A. et al. MiR-200a Regulates CDK4/6 Inhibitor Effect by Targeting CDK6 in Metastatic Melanoma. J Invest Dermato/ 137, 1955-1964, doi:10.1016/j.jid.2017.03.039 (2017).

42. Inoue, K. \& Fry, E. A. Aberrant expression of cyclin D1 in cancer. Sign Transduct Insights 4, 1-13, doi:10.4137/STI.S30306 (2015).

43. Fry, D. W. et al. Specific inhibition of cyclin-dependent kinase 4/6 by PD 0332991 and associated antitumor activity in human tumor xenografts. Mol Cancer Ther 3, 1427-1438 (2004).

44. Borysov, S. I. \& Guadagno, T. M. A novel role for Cdk1/cyclin B in regulating B-raf activation at mitosis. Mol Biol Cell 19, 2907-2915, doi:10.1091/mbc.E07-07-0679 (2008).

45. Keenan, S. M., Bellone, C. \& Baldassare, J. J. Cyclin-dependent kinase 2 nucleocytoplasmic translocation is regulated by extracellular regulated kinase. J Biol Chem 276, 22404-22409, doi:10.1074/jbc.M100409200 (2001).

46. Lunn, C. L., Chrivia, J. C. \& Baldassare, J. J. Activation of Cdk2/Cyclin E complexes is dependent on the origin of replication licensing factor Cdc6 in mammalian cells. Cell Cycle 9, 4533-4541, doi:10.4161/cc.9.22.13789 (2010).

47. Duong, M. T. et al. Hbo1 is a cyclin E/CDK2 substrate that enriches breast cancer stem-like cells. Cancer Res 73, 5556-5568, doi:10.1158/0008-5472.CAN-13-0013 (2013).

48. Neganova, I. et al. CDK1 plays an important role in the maintenance of pluripotency and genomic stability in human pluripotent stem cells. Cell Death Dis 5, e1508, doi:10.1038/cddis.2014.464 (2014).

49. Peyressatre, M., Prevel, C., Pellerano, M. \& Morris, M. C. Targeting cyclin-dependent kinases in human cancers: from small molecules to Peptide inhibitors. Cancers (Basel) 7, 179-237, doi:10.3390/cancers7010179 (2015). 
50. Parry, D. et al. Dinaciclib (SCH 727965), a novel and potent cyclin-dependent kinase inhibitor. $\mathrm{Mol}$ Cancer Ther 9, 2344-2353, doi:10.1158/1535-7163.MCT-10-0324 (2010).

51. Meijer, L. et al. Biochemical and cellular effects of roscovitine, a potent and selective inhibitor of the cyclin-dependent kinases cdc2, cdk2 and cdk5. Eur J Biochem 243, 527-536, doi:10.1111/j.14321033.1997.t01-2-00527.x (1997).

52. Solomon, M. J., Lee, T. \& Kirschner, M. W. Role of phosphorylation in p34cdc2 activation: identification of an activating kinase. Mol Biol Cel/ 3, 13-27, doi:10.1091/mbc.3.1.13 (1992).

53. Yuzwa, S. A. et al. A potent mechanism-inspired O-GlcNAcase inhibitor that blocks phosphorylation of tau in vivo. Nat Chem Bio/4, 483-490, doi:10.1038/nchembio. 96 (2008).

54. Afify, S. M. \& Seno, M. Conversion of Stem Cells to Cancer Stem Cells: Undercurrent of Cancer Initiation. Cancers (Basel) 11, doi:10.3390/cancers11030345 (2019).

55. Ayob, A. Z. \& Ramasamy, T. S. Cancer stem cells as key drivers of tumour progression. J Biomed Sci 25, 20, doi:10.1186/s12929-018-0426-4 (2018).

56. Jin, X., Jin, X. \& Kim, H. Cancer stem cells and differentiation therapy. Tumour Bio/39, 1010428317729933, doi:10.1177/1010428317729933 (2017).

57. Kreso, A. \& Dick, J. E. Evolution of the cancer stem cell model. Cell Stem Cell 14, 275-291, doi:10.1016/j.stem.2014.02.006 (2014).

58. Plaks, V., Kong, N. \& Werb, Z. The cancer stem cell niche: how essential is the niche in regulating stemness of tumor cells? Cell Stem Cell 16, 225-238, doi:10.1016/j.stem.2015.02.015 (2015).

59. Friedmann-Morvinski, D. \& Verma, I. M. Dedifferentiation and reprogramming: origins of cancer stem cells. EMBO Rep 15, 244-253, doi:10.1002/embr.201338254 (2014).

60. Takahashi, K. \& Yamanaka, S. A decade of transcription factor-mediated reprogramming to pluripotency. Nat Rev Mol Cell Bio/ 17, 183-193, doi:10.1038/nrm.2016.8 (2016).

61. Heo, J. et al. Phosphorylation of TFCP2L1 by CDK1 is required for stem cell pluripotency and bladder carcinogenesis. EMBO Mol Med 12, e10880, doi:10.15252/emmm.201910880 (2020).

62. Yeh, I. J. et al. Phosphorylation of the histone demethylase KDM5B and regulation of the phenotype of triple negative breast cancer. Sci Rep 9, 17663, doi:10.1038/s41598-019-54184-0 (2019).

63. McClain, D. A. \& Crook, E. D. Hexosamines and insulin resistance. Diabetes 45, 1003-1009 (1996).

64. Kimmelman, A. C. Metabolic Dependencies in RAS-Driven Cancers. Clin Cancer Res 21, 1828-1834, doi:10.1158/1078-0432.CCR-14-2425 (2015).

65. Myers, S. A. et al. SOX2 O-GlcNAcylation alters its protein-protein interactions and genomic occupancy to modulate gene expression in pluripotent cells. Elife 5, e10647, doi:10.7554/eLife.10647 (2016).

66. Lane, D. P. Cancer. p53, guardian of the genome. Nature 358, 15-16, doi:10.1038/358015a0 (1992).

67. Lowe, S. W., Cepero, E. \& Evan, G. Intrinsic tumour suppression. Nature 432, 307-315, doi:10.1038/nature03098 (2004). 
68. Vousden, K. H. \& Ryan, K. M. p53 and metabolism. Nat Rev Cancer 9, 691-700, doi:10.1038/nrc2715 (2009).

69. Li, T. et al. Tumor suppression in the absence of p53-mediated cell-cycle arrest, apoptosis, and senescence. Cell 149, 1269-1283, doi:10.1016/j.cell.2012.04.026 (2012).

70. Bensaad, K. et al. TIGAR, a p53-inducible regulator of glycolysis and apoptosis. Cel/ 126, 107-120, doi:10.1016/j.cell.2006.05.036 (2006).

71. Mosteiro, L. et al. Tissue damage and senescence provide critical signals for cellular reprogramming in vivo. Science 354, doi:10.1126/science.aaf4445 (2016).

72. Tanimura, A., Nakazato, A. \& Tanaka, N. MYD88 signals induce tumour-initiating cell generation through the NF-kappaB-HIF-1 alpha activation cascade. Sci Rep 11, 3991, doi:10.1038/s41598-02183603-4 (2021).

\section{Figures}
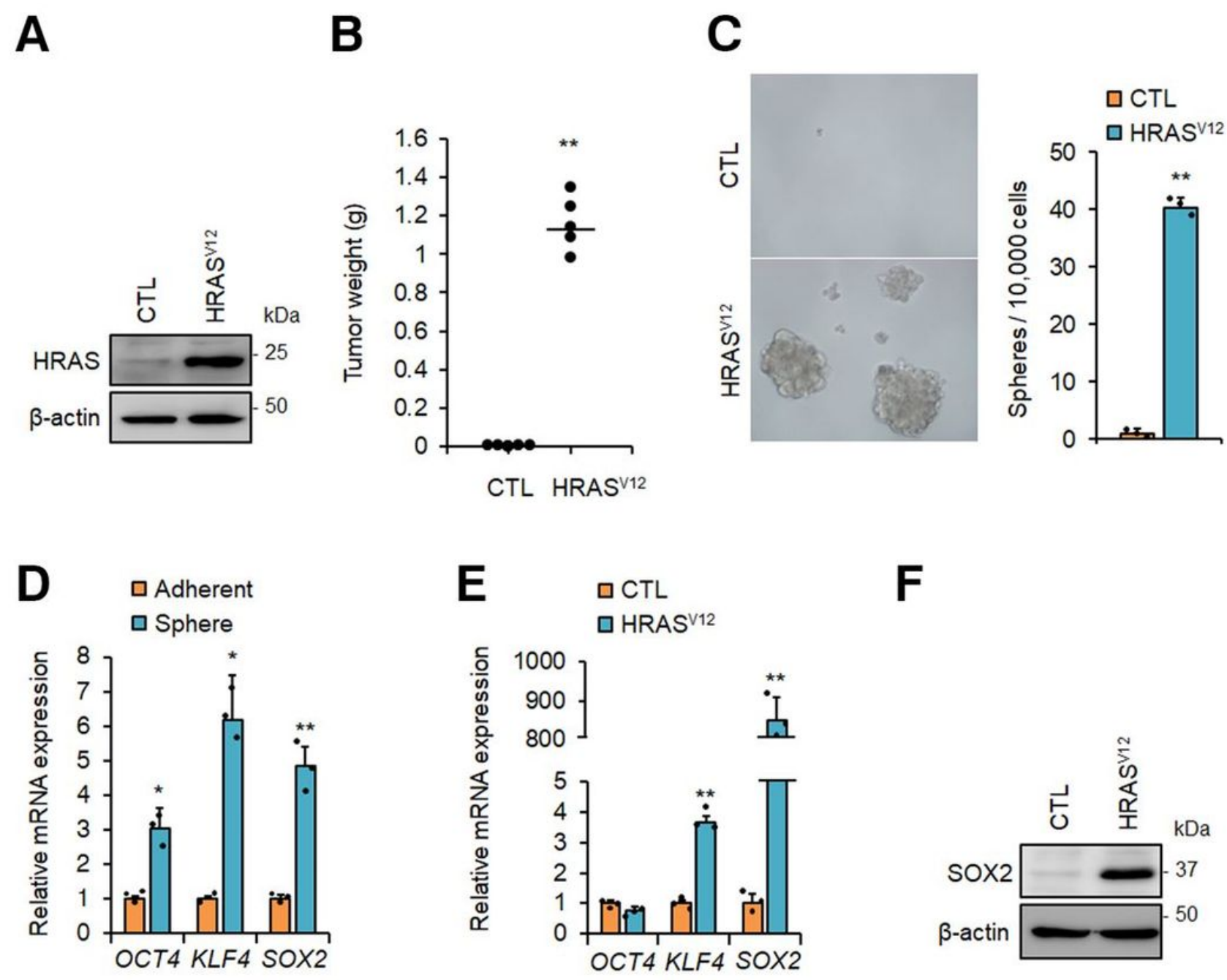

Figure 1 
Oncogenic RAS induces SOX2-initiated CSC generation. A, Vector only or the HRASV12 mutant was transduced into p53-/- mouse embryonic fibroblasts (MEFs) by retroviral infection for 2 days, and cells were selected with hygromycin for 3 days. Expression of the HRASV12 mutant was confirmed by immunoblotting analysis. B, p53-/- MEFs (CTL) or HRASV12-expressing p53-/- MEFs $(1 \times 105)$ were subcutaneously injected into immunodeficient mice ( $n=5$ per group). Tumour weights were measured 3 weeks after injection. ${ }^{*} \mathrm{P}<0.01$. C, Sphere formation by $\mathrm{p} 53-/-\mathrm{MEFs}(\mathrm{CTL})$ or HRASV12-expressing p53-/- MEFs. Representative images are shown on the left; the graph on the right shows quantification of the numbers of spheres counted per 10,000 cells. $* * P<0.01$. D, qPCR analysis of the levels of the stem cell marker genes OCT4, KLF4, and SOX2 in adherent cells or spheres from HRASV12-expressing p53-/MEFs. The mRNA expression levels were normalised to $\beta$-actin. ${ }^{\star} \mathrm{P}<0.05$, ${ }^{*} \mathrm{P}<0.01$. E, mRNA expression levels of stem cell marker genes in p53-/- MEFs (CTL) and HRASV12-expressing p53-/- MEFs. $\beta$-actin mRNA was used for normalisation. ${ }^{* * P}<0.01$. F, Immunoblot of SOX2 protein expression in $\mathrm{p} 53-/-\mathrm{MEFs}$ and HRASV12-expressing p53-/-MEFs. B-E: Data are presented as the means \pm SD of three independent experiments. Statistical analysis was performed with Student's t-test. Uncropped blot images are presented in Supplementary Fig. S5.

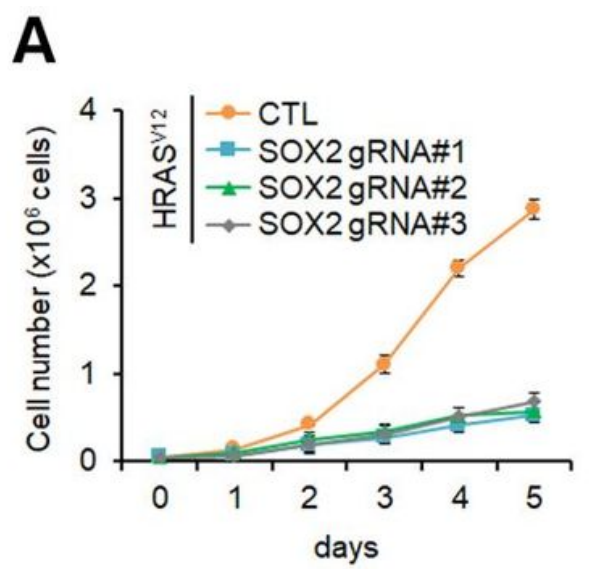

B

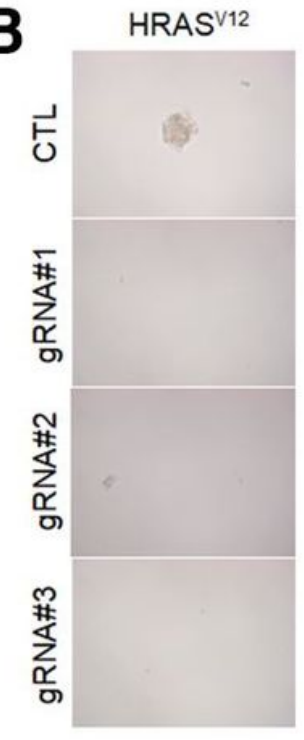

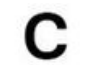

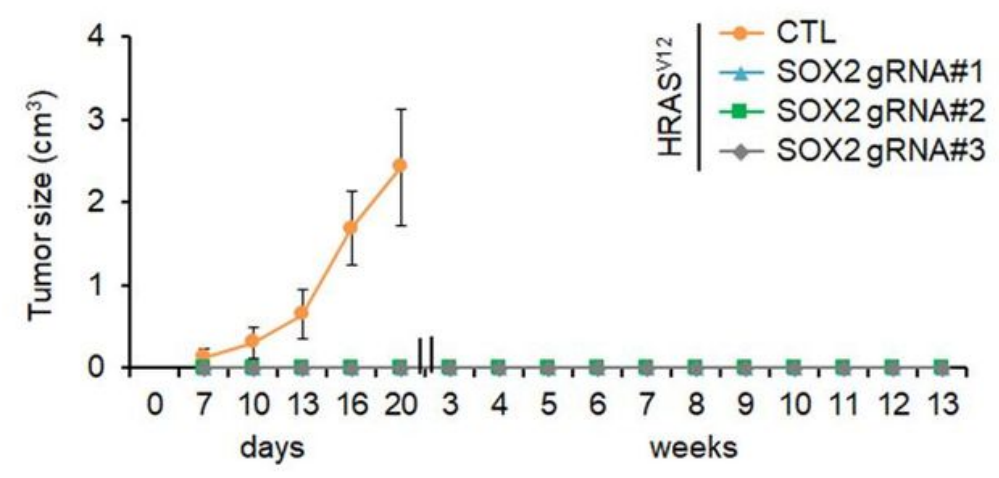

D
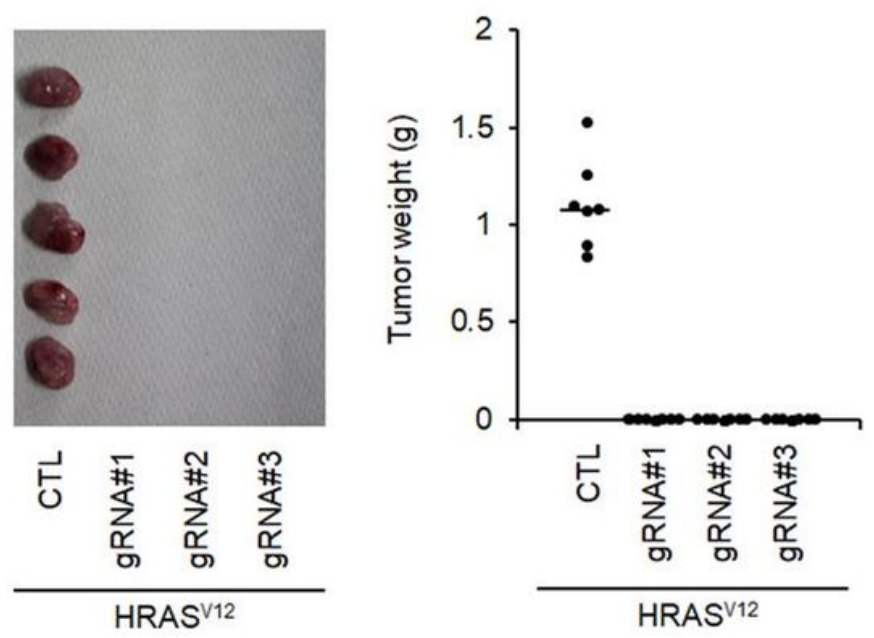

Figure 2 
SOX2 expression is essential for HRASV12-induced development of CSC properties. A, SOX2 sgRNAs were co-expressed with CRISPR and Cas9 proteins in HRASV12-expressing p53-/- mouse embryonic fibroblasts (MEFs) by lentiviral infection for 2 days, and cells were selected with puromycin for 3 days. Growth curves for HRASV12-expressing p53-/- MEFs containing each SOX2 gRNA or vehicle (CTL) are shown. B, Sphere formation of SOX2-deficient HRASV12-expressing p53-/- MEFs. Representative images are shown on the left and quantification is shown on the right. ND, not detected. $C$ and $D$, Immunodeficient mice $(n=5)$ were subcutaneously injected with $1 \times 105$ of HRASV12-expressing p53-/MEFs containing each SOX2 gRNA or vehicle (CTL). C, Tumour sizes were measured every 3 days for 3 weeks and each week thereafter. D, Tumours and tumour weights at 3 weeks post-injection for each mouse are indicated. 

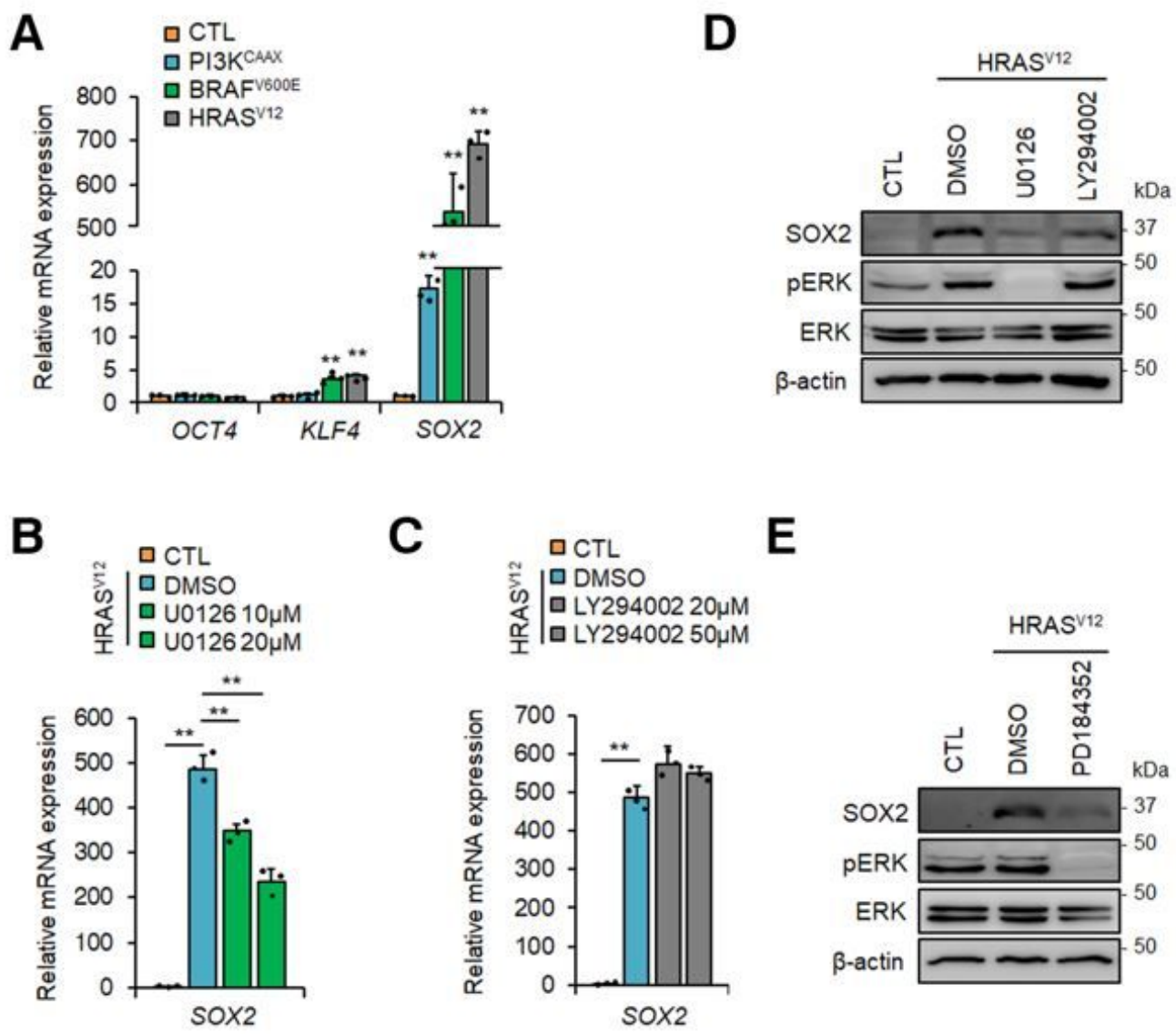

E

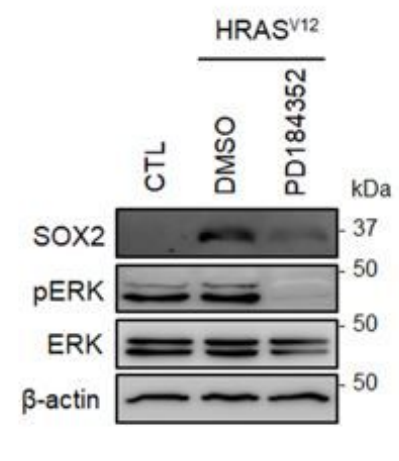

$\mathbf{F}$

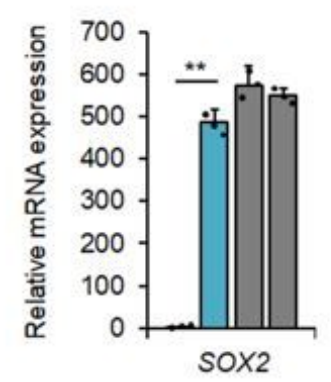

\section{G}
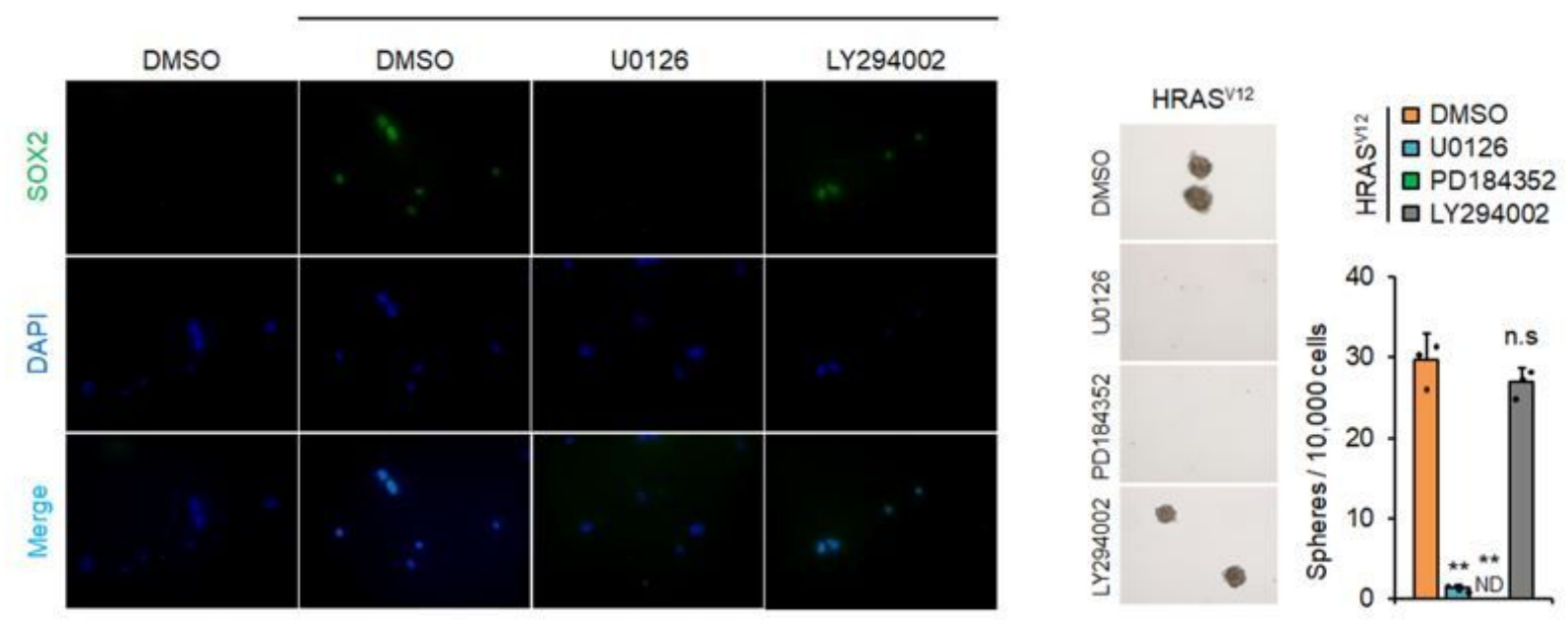

\section{Figure 3}

SOX2 expression is induced by the RAF-MAPK pathway in HRASV12-expressing p53-/- mouse embryonic fibroblasts (MEFs). A, Expression levels of OCT4, KLF4, and SOX2 mRNA in PI3KCAAX-, BRAFV600E-, or HRASV12-expressing p53-/- MEFs. The levels of stem cell marker genes in these cells were compared with those in p53-/- MEFs (CTL). ${ }^{*} \mathrm{P}<0.01$. B and C, qPCR analysis of SOX2 mRNA in HRASV12-expressing p53-/- MEFs treated with vehicle, U0126 (10, $20 \mu \mathrm{M})$, or LY294002 (20, $50 \mu \mathrm{M})$ for 
24 h. ${ }^{* * P}<0.01$. D, Immunoblotting analysis of SOX2 expression in HRASV12-expressing p53-/- MEFs treated with vehicle, U0126 $(10 \mu \mathrm{M})$, or LY294002 $(20 \mu \mathrm{M})$ for $24 \mathrm{~h}$. E, Expression levels of SOX2 protein in HRASV12-expressing p53-/- MEFs treated with vehicle or PD184352 (1 $\mu \mathrm{M})$ for $24 \mathrm{~h}$. F, HRASV12expressing p53-/- MEFs were stimulated with vehicle, U0126 $(10 \mu \mathrm{M})$, or LY294002 (20 $\mu \mathrm{M})$ for $24 \mathrm{~h}$. The cells were stained with anti-SOX2 antibody (Green) and DAPI (Blue) and visualised by a fluorescence microscope under 200x magnification. G, Sphere formation in HRASV12-expressing p53-/- MEFs cultured with vehicle (DMSO), U0126 (10 $\mu \mathrm{M})$, PD184352 $(1 \mu \mathrm{M})$, or LY294002 $(20 \mu \mathrm{M})$ for 7 days. The numbers of spheres in each group were compared. ND, not detected; $n$. S., not significant. ${ }^{*} P<0.01$. A-C and G: Data are presented as the means \pm SD of three independent experiments. Statistical analysis was performed with Student's t-tests. Uncropped blot images are presented in Supplementary Fig. S5.

A
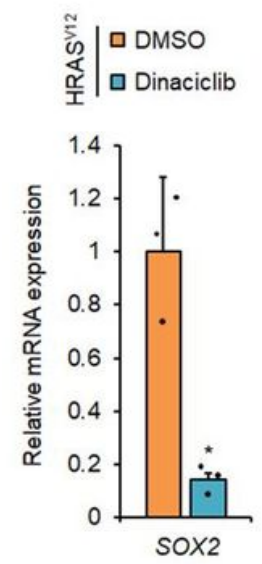

E

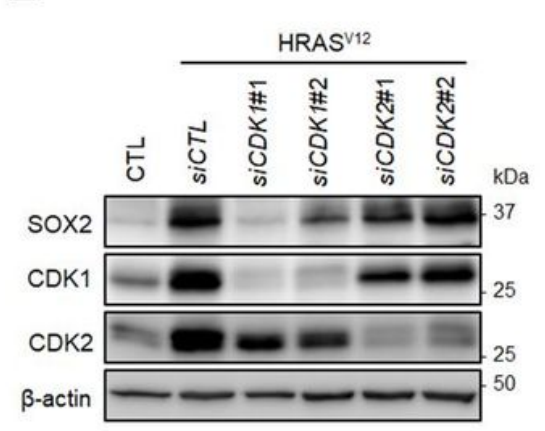

B

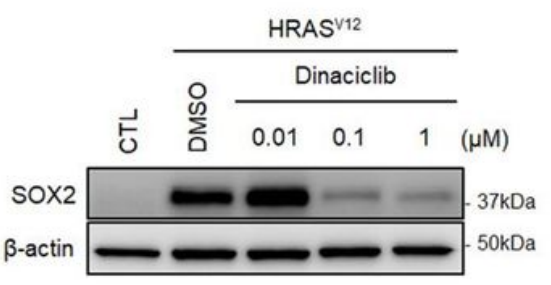

C

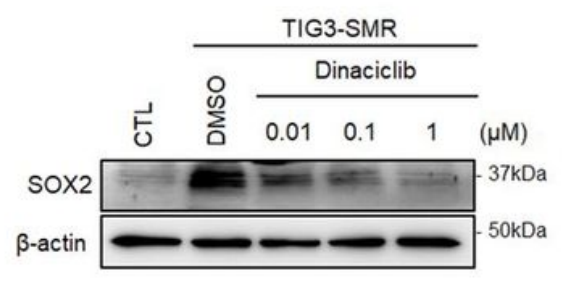

F
D

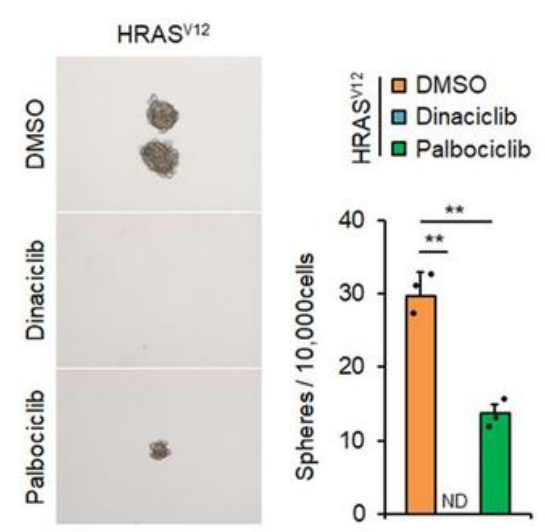


HRASV12-expressing p53-/- MEFs following knockdown of CDK1 or CDK2 by siRNAs. F, qPCR analysis of SOX2 mRNA expression in HCT116, SW480, DLD1, H460, and A549 cancer cell lines treated with dinaciclib $(0.1 \mu \mathrm{M})$ for $24 \mathrm{~h} .{ }^{*} \mathrm{P}<0.05,{ }^{*} \mathrm{P}<0.01$. G, Sphere formation assay of cancer cells in the presence or absence of dinaciclib $(0.1 \mu \mathrm{M})$ for 7 days. ${ }^{* *} \mathrm{P}<0.01$. $A, D, F$, and $G$ : Data are presented as the means \pm SD of three independent experiments. Statistical analysis was performed with Student's t-tests. Uncropped blot images are presented in Supplementary Fig. S5.
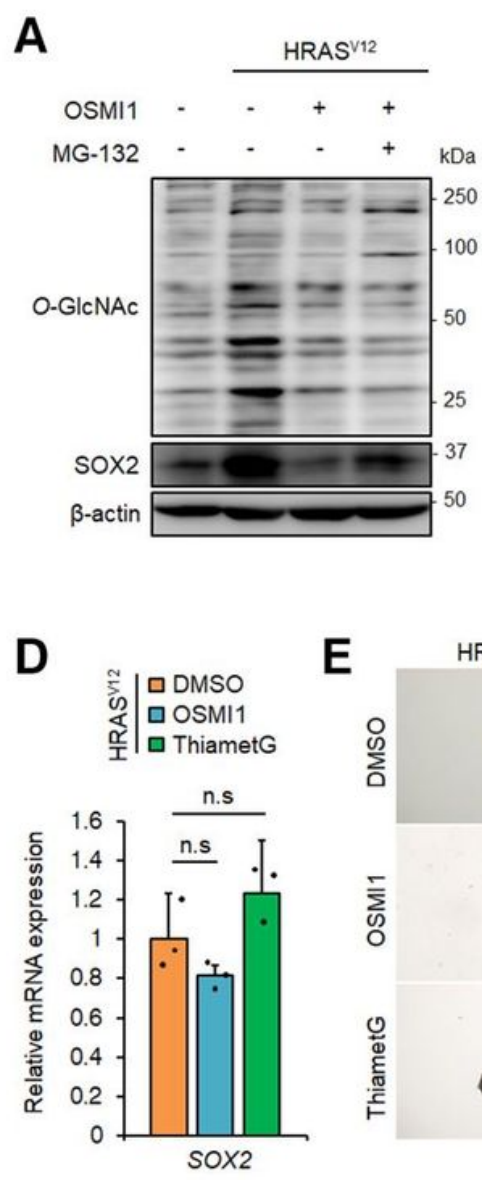
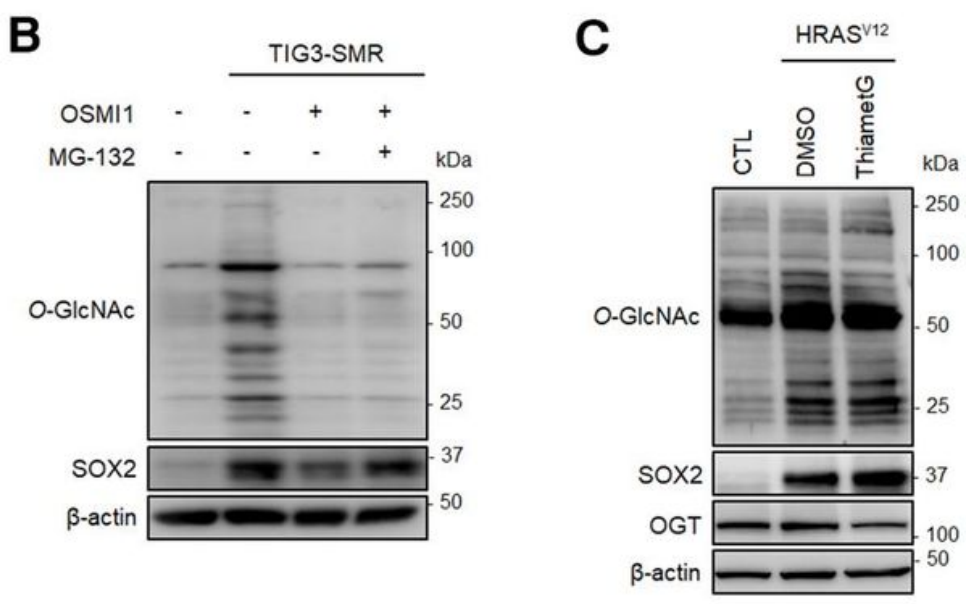
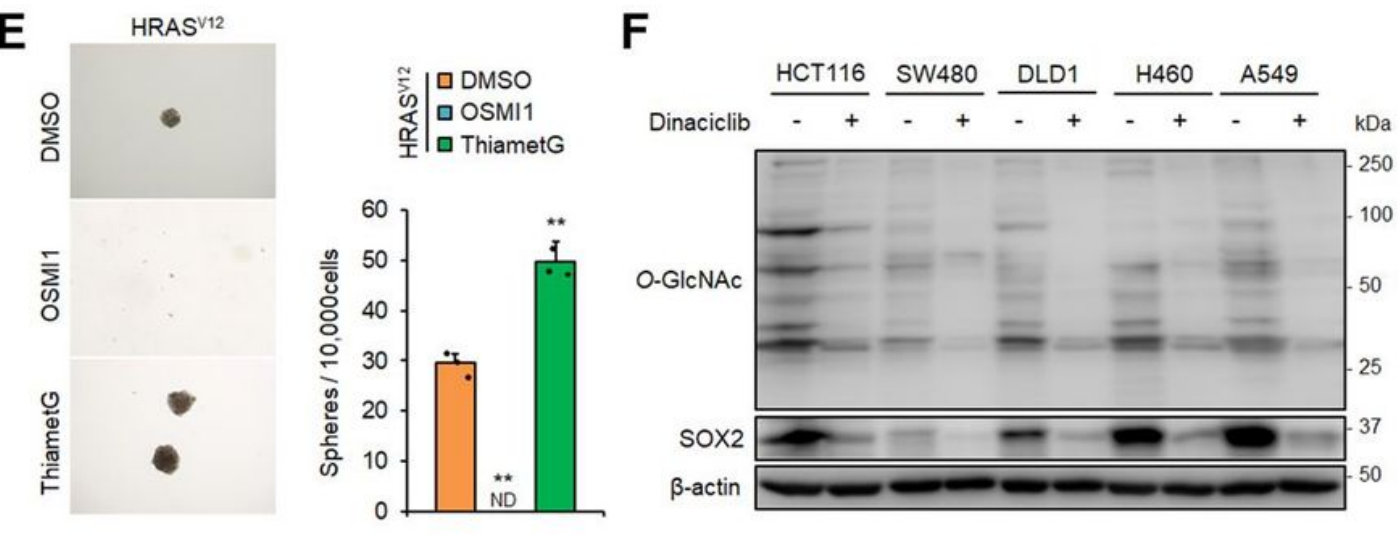

\section{Figure 5}

Enhanced O-GIcNAc modification induced by the RAS/MAPK/CDK1 pathway is required for SOX2 protein expression and generation of CSCs. A and B, Immunoblot analysis of O-GlcNAc-modified proteins and SOX2 expression in HRASV12-expressing p53-/- mouse embryonic fibroblasts (MEFs) (A) and TIG-3SMR cells $(B)$ treated with vehicle or OSMI1 $(50 \mu \mathrm{M})$ for $24 \mathrm{~h}$ in the presence or absence of MG-132 (20 $\mu \mathrm{M})$ for $6 \mathrm{~h}$. C, Immunoblots of O-GIcNAc modified proteins and SOX2 expression in HRASV12-expressing p53-/- MEFs treated with vehicle or thiamet $\mathrm{G}(10 \mu \mathrm{M})$ for $24 \mathrm{~h}$. D, Expression levels of SOX2 mRNA in HRASV12-expressing p53-/- MEFs cells treated with OSMI1 $(50 \mu \mathrm{M})$ or thiamet G $(10 \mu \mathrm{M})$ for $24 \mathrm{~h}$. n. s., not significant. E, Sphere formation assay of HRASV12-expressing p53-/- MEFs cultured with OSMI1 (50 $\mu \mathrm{M})$ or thiamet $\mathrm{G}(10 \mu \mathrm{M})$ for 7 days. Representative images are shown on the left; the graph on the right shows quantification of the numbers of spheres counted per 10,000 cells. ND, not detected. ${ }^{\star \star} P<0.01$. F, Immunoblot analysis of SOX2 and O-GIcNAc levels in HCT116, SW480, DLD1, H460, and A549 cancer cells treated with dinaciclib $(0.1 \mu \mathrm{M})$ for $24 \mathrm{~h}$. Data for $\mathrm{D}$ and $\mathrm{E}$ are presented as the means $\pm \mathrm{SD}$ of three 
independent experiments. Statistical analysis was performed with Student's t-tests. Uncropped blot images are presented in Supplementary Fig. S5.

\section{Supplementary Files}

This is a list of supplementary files associated with this preprint. Click to download.

- Shimizu.Suppllnfo10.29.pdf 